Supporting Information for

\title{
Stereochemical Recognition of Helicenes on Metal Surfaces
}

Karl-Heinz Ernst ${ }^{*, \delta, \dagger}$

${ }^{\S}$ Nanoscale Materials Science, Empa, Swiss Federal Laboratories for Materials Science and Technology, Überlandstrasse 129, CH-8600 Dübendorf, Switzerland

${ }^{\dagger}$ Department of Chemistry, University of Zurich, Winterthurerstrasse 190, CH-8057

Zürich, Switzerland

*E-mail: karl-heinz.ernst@empa.ch

Content:

Table S1: Electrical STM parameters of all STM images of the publication (page S2).

Table S2: Structural Parameters of [7]H Adsorbate Lattices (page S3). 
Table S1. Electrical parameters of STM images

\begin{tabular}{|c|c|c|}
\hline Figure & Bias voltage $[\mathrm{V}]$ & Tunneling current [pA] \\
\hline $2 \mathrm{a}$ & -2.90 & 43 \\
\hline $2 b$ & 2.90 & 21 \\
\hline $2 c$ & 2.05 & 19 \\
\hline $2 \mathrm{~d}$ & 2.73 & 26 \\
\hline $2 \mathrm{~g}$ & 2.73 & 20 \\
\hline $3 \mathrm{a}$ & 2.61 & 20 \\
\hline $3 b$ & -1.67 & 1280 \\
\hline $4 a$ & -2.73 & 40 \\
\hline $4 \mathrm{~b}$ & -3.08 & 10 \\
\hline 4b-green & 2.61 & 42 \\
\hline 4b-blue & 2.73 & 42 \\
\hline $4 \mathrm{c}$ & -1.19 & 179 \\
\hline $4 d$ & -2.84 & 31 \\
\hline 4d-insets & 2.84 & 19 \\
\hline $4 \mathrm{e}$ & 2.84 & 19 \\
\hline $4 \mathrm{f}$ & 2.73 & 27 \\
\hline $4 \mathrm{f}$-inset & 2.84 & 19 \\
\hline $4 \mathrm{~g}$ & -2.78 & 20 \\
\hline $5 \mathrm{a}$ & 0.50 & 20 \\
\hline $5 b$ & 1.6 & 10 \\
\hline $5 c$ & 0.50 & 20 \\
\hline $6 a$ & -2.73 & 25 \\
\hline $6 b, 6 c, 6 d$ & 2.05 & 28 \\
\hline $7 a$ & 2.56 & 26 \\
\hline $7 \mathrm{~b}$ & 2.56 & 23 \\
\hline $8 \mathrm{a}$ & -2.73 & 66 \\
\hline $9 a$ & 2.72 & 20 \\
\hline $9 b$ & 2.96 & 19 \\
\hline $9 c$ & -2.56 & 22 \\
\hline $9 \mathrm{~d}$ & -2.36 & 55 \\
\hline $9 \mathrm{e}$ & 2.67 & 25 \\
\hline $9 f$ & 2.89 & 16 \\
\hline $9 g$ & 3.02 & 14 \\
\hline
\end{tabular}


Table S2. Structural Parameters of [7]H and db-[5]H Adsorbate Lattices

\begin{tabular}{|c|c|c|c|c|}
\hline Adsorbate system & $\begin{array}{c}\text { Matrix } \\
\text { notation }\end{array}$ & $\begin{array}{l}\text { Tilt angle } \\
\text { w.r.t. }[1 \overline{1} 0] \\
\text { for }[01 \overline{1}] \\
\text { for }(100) \\
\text { faces\} }\end{array}$ & $\begin{array}{l}\text { Molecules } \\
\text { per unit } \\
\text { cell }\end{array}$ & $\begin{array}{l}\text { Covered } \\
\text { area per } \\
\text { molecule }\end{array}$ \\
\hline$(P)-[7] \mathrm{H} / \mathrm{Cu}(111)$ & $\left(\begin{array}{cc}12 & -2 \\
2 & 14\end{array}\right)$ & $7.6^{\circ}$ & 9 & $1.08 \mathrm{~nm}^{2}$ \\
\hline$(M)-[7] \mathrm{H} / \mathrm{Cu}(111)$ & $\left(\begin{array}{cc}8 & 2 \\
-2 & 6\end{array}\right)$ & $13.3^{\circ}$ & 3 & $0.98 \mathrm{~nm}^{2}$ \\
\hline$(M)-[7] \mathrm{H} / \operatorname{Ag}(111)$ & $\left(\begin{array}{cc}6 & -1 \\
1 & 7\end{array}\right)$ & $10^{\circ}$ & 3 & $1.03 \mathrm{~nm}^{2}$ \\
\hline$(M)-[7] \mathrm{H} / \mathrm{Au}(111)$ & $\left(\begin{array}{cc}6 & -1 \\
1 & 7\end{array}\right)$ & $10^{\circ}$ & 3 & $1.03 \mathrm{~nm}^{2}$ \\
\hline$(M)-[7] \mathrm{H} / \mathrm{Au}(111)$ & $\left(\begin{array}{cc}6 & -2 \\
1 & 10\end{array}\right)$ & $5^{\circ}$ & 4 & $1.12 \mathrm{~nm}^{2}$ \\
\hline$(M)-[7] \mathrm{H} / \mathrm{Cu}(100)$ & $\left(\begin{array}{cc}8 & 2 \\
-2 & 8\end{array}\right)$ & $14.5^{\circ}$ & 4 & $1.10 \mathrm{~nm}^{2}$ \\
\hline$(M)-[7] \mathrm{H} / \operatorname{Ag}(100)$ & $\left(\begin{array}{cc}7 & 2 \\
-2 & 7\end{array}\right)$ & $14.5^{\circ}$ & 4 & $1.10 \mathrm{~nm}^{2}$ \\
\hline$r a c-[7] \mathrm{H} / \mathrm{Cu}(111)$ & $\left(\begin{array}{cc}4 & -1 \\
5 & 8\end{array}\right)$ & $10.9^{\circ}$ & 2 & $1.04 \mathrm{~nm}^{2}$ \\
\hline rac-[7] $\mathrm{H} / \mathrm{Cu}(111)$ & $\left(\begin{array}{ll}5 & 2 \\
1 & 8\end{array}\right)$ & $23.4^{\circ}$ & 2 & $1.07 \mathrm{~nm}^{2}$ \\
\hline$r a c-[7] \mathrm{H} / \operatorname{Ag}(111)$ & $\left(\begin{array}{ll}4 & 0 \\
1 & 7\end{array}\right)$ & $0^{\circ}$ & 2 & $1.04 \mathrm{~nm}^{2}$ \\
\hline rac-[7]H/Au(111) & - & $0^{\circ}$ & - & - \\
\hline$r a c-[7] \mathrm{H} / \mathrm{Cu}(100)$ & $\begin{array}{c}\text { conglomerate, } \\
\text { see }(M)-[7] \mathrm{H}\end{array}$ & $14.5^{\circ}$ & 4 & $1.10 \mathrm{~nm}^{2}$ \\
\hline$r a c-[7] \mathrm{H} / \operatorname{Ag}(100)$ & $\left(\begin{array}{ll}4 & 0 \\
0 & 7\end{array}\right)$ & $0^{\circ}$ & 2 & $1.17 \mathrm{~nm}^{2}$ \\
\hline$r a c-\mathrm{db}[5] \mathrm{H} / \mathrm{Au}(111)$ & $\left(\begin{array}{cc}6 & 2 \\
1 & 13\end{array}\right)$ & $12^{\circ}$ & 4 & $1.37 \mathrm{~nm}^{2}$ \\
\hline
\end{tabular}

